\title{
Jinmaitong decreases sciatic nerve DNA oxidative damage and apoptosis in a streptozotocin-induced diabetic rat model
}

\author{
DE-HAI YIN ${ }^{1}$, XIAO-CHUN LIANG ${ }^{1}$, LI ZHAO $^{2}$, HONG ZHANG $^{3}$, \\ QING SUN ${ }^{1}$, PU-YAN WANG ${ }^{1}$ and LIAN-QING SUN ${ }^{1}$ \\ ${ }^{1}$ Department of Traditional Chinese Medicine, Peking Union Medical College Hospital, Beijing 100032; \\ ${ }^{2}$ Department of Nephrology and Endocrinology, Dongzhimen Hospital Affiliated to Beijing University of Chinese Medicine, \\ Beijing 100700; ${ }^{3}$ Cell Resource Center, School of Basic Medicine, Peking Union Medical College, Beijing 100005, P.R. China
}

Received June 17, 2014; Accepted February 16, 2015

DOI: $10.3892 / \mathrm{etm} .2015 .2543$

\begin{abstract}
Diabetic peripheral neuropathy (DPN) is a common chronic complication of diabetes. Jinmaitong (JMT), a Traditional Chinese Medicine, improves certain symptoms of DPN, such as limb pain and numbness. The aim of the present study was to investigate the effects of JMT on DNA oxidative damage and apoptosis in the sciatic nerve of diabetic rats. The rats were divided into a normal and a diabetic group. Diabetes was induced using streptozotocin $(60 \mathrm{mg} / \mathrm{kg})$. The diabetic model (DM) rats received vitamin C $(0.05 \mathrm{~g} / \mathrm{kg} / \mathrm{day})$ or JMT [low-dosage (L), $0.44 \mathrm{~g} / \mathrm{kg} / \mathrm{day}$; medium-dosage (M), $0.88 \mathrm{~g} / \mathrm{kg} /$ day or high-dosage $(\mathrm{H}), 1.75 \mathrm{~g} / \mathrm{kg} / \mathrm{day}]$. After 16 weeks, the mechanical pain threshold of the rats was evaluated. The expression of 8-hydroxy-deoxyguanosine (8-OHdG), nicotinamide adenine dinucleotide phosphate (NADPH) oxidase p22 phox, B-cell lymphoma 2 (Bcl-2), caspase 3 and cleaved-poly(ADP-ribose) polymerase 1 (PARP-1) in the sciatic nerve tissues was measured using the reverse transcription-quantitative polymerase chain reaction, immunohistochemistry and western blotting. JMT had no effect on body weight and fasting blood glucose levels. Following treatment, the rats in the JMT groups had an improved pain threshold compared with the DM controls (JMT-L, 52.9 $\pm 6.5 \mathrm{~g}$; JMT-M, 74.7 $\pm 9.3 \mathrm{~g}$; and JMT-H, 61.7 $\pm 2.0 \mathrm{~g}$ vs. DM control, $35.32 \pm 12.06 \mathrm{~g}$; all $\mathrm{P}<0.01)$, while the threshold in the JMT-M rats was similar to that of normal controls $(\mathrm{P}>0.05)$. 8-OHdG and NADPH oxidase $\mathrm{p} 22^{\text {phox }}$ expression was significantly decreased in the three JMT groups compared with that in the DM controls (all $\mathrm{P}<0.05)$. Following JMT treatment, Bcl-2 levels were increased, while caspase 3 and cleaved-PARP-1 levels were decreased compared with those in the DM controls
\end{abstract}

Correspondence to: Dr Xiao-Chun Liang, Department of Traditional Chinese Medicine, Peking Union Medical College Hospital, 41 Damucang Hutong, Beijing 100032, P.R. China E-mail: liangxcmedsci@163.com

Key words: diabetic peripheral neuropathy, Jinmaitong capsule, apoptosis, DNA oxidative damage, sciatic nerve (all $\mathrm{P}<0.01$ ). In conclusion, JMT may reduce DNA oxidative damage to the sciatic nerve in diabetic rats, as well as regulate genes involved in peripheral neuronal cell apoptosis, suggesting that JMT could be used to prevent or treat DPN in diabetic patients.

\section{Introduction}

Diabetic peripheral neuropathy (DPN) is one of the most common chronic complications of diabetes, inducing disability and decreased quality of life $(1,2)$. Although reports of the prevalence of DPN vary substantially, depending on the diagnostic criteria and investigation depth, the majority of studies suggest that $\sim 50 \%$ of all diabetic patients are likely to develop DPN (2-4), and that the duration and severity of the diabetes are the major risk factors (5). Early symptoms include loss of vibratory perception, altered proprioception and impairment of pain, touch and temperature perception, ultimately leading to a range of complications (foot ulcers, foot and joint diseases and amputation) $(6,7)$. Current treatments for DPN focus on glycemic control, foot care and the treatment of pain $(3,4,8)$; therefore, these treatments mainly focus on the symptoms of DPN, rather than on its causes.

DPN is caused by an imbalance between nerve damage and repair, and is correlated with the apoptosis of peripheral nerve cells $(9,10)$. This imbalance is mainly fed by the oxidative stress cycle, which induces important damage to nerve cells (11-14). Oxidative stress leads to DNA oxidative damage in these cells, which activates apoptosis (14-16).

Traditional Chinese Medicine has long recognized that DPN could be efficiently treated with medicines such as Jinmaitong (JMT) (17). JMT capsules can improve DPN symptoms, such as limb pain and numbness, by increasing the nerve conduction velocity, and by improving glucose and lipid metabolism (17-19). Studies have shown that JMT can reduce diabetic damage to the sciatic nerve by reducing the accumulation of sorbitol, by upregulating the expression of ciliary neurotrophic factor (CNTF), by reducing the formation of advanced glycosylation end products (AGEs) and by downregulating the expression of AGE receptors $(18,19)$. In addition, preliminary studies have shown that JMT can increase superoxide dismutase activity in diabetic rats, and reduce lipid 
peroxidation $(18,19)$. Despite this, the specific mechanisms underlying the action of JMT are poorly understood.

The aim of the present study was to investigate the effects of JMT on specific markers of DNA oxidative damage [8-hydroxy-deoxyguanosine (8-OHdG) and nicotinamide adenine dinucleotide phosphate (NADPH) oxidase], as well as on factors involved in apoptosis, such as B-cell lymphoma 2 (Bcl-2), caspase 3 and cleaved-poly(ADP-ribose) polymerase 1 (PARP-1), in the sciatic nerve of a diabetic rat model.

\section{Materials and methods}

Animals. Male six-week-old Sprague Dawley rats ( $\mathrm{n}=86$; body weight, 231-267 g) were provided by Vital River Laboratory Animal Technology Co., Ltd. [Beijing, China; certificate no. SCXK (Beijing) 2007-0001], and were bred in the Experimental Animal Center (specific pathogen-free level) of the Peking Union Medical College Hospital (Beijing, China). Rats were acclimated for two week before the commencement of the experiment. This study was carried out in strict accordance with the recommendations of the Guide for the Care and Use of Laboratory Animals of the National Institutes of Health. The protocol was approved by the Institutional Animal Care and Use Committee of the Peking Union Medical College Hospital.

Diabetic rat model and grouping. The diabetic rat model was induced as previously described (20). After a two-week adaptation period, rats were randomly divided into the normal and diabetic model (DM) groups. After a 12-h fast, a $0.45 \%$ streptozotocin $(60 \mathrm{mg} / \mathrm{kg}$ ) solution (Sigma, St. Louis, MO, USA) prepared in $0.1 \mathrm{~mol} / 1$ citrate buffer $(\mathrm{pH} 4.5)$, was intraperitoneally injected in rats of the DM group, while rats in the normal group were injected with the same volume of $0.1 \mathrm{~mol} / \mathrm{l}$ citrate buffer. Blood glucose was measured from the tail tip $72 \mathrm{~h}$ later using a blood glucose meter (MediSense ${ }^{\circledR}$ Optium $^{\text {TM }}$; Abbott Laboratories, Chicago, IL, USA). Blood glucose levels $\geq 16.7 \mathrm{mmol} / 1$ indicated model success, and the success rate was $92.1 \%$ (70/76). Consequently, 70 successfully modeled diabetic rats were randomly divided into five groups (n=14/group): DM control, JMT low-dosage (JMT-L), JMT medium-dosage (JMT-M), JMT high-dosage (JMT-H) and vitamin $\mathrm{C}$ (VC). The normal control group (Con) included 10 rats with blood glucose levels $<7.0 \mathrm{mmol} / \mathrm{l}$. A rat of the JMT-L group died in the initial stage of modeling. Twelve weeks after modeling, eight rats died: three in the DM group, one in each of the JMT-L, -M and $-\mathrm{H}$ groups and two in the VC group. Sixteen weeks after modeling, one rat in the JMT-H group died from unsuccessful gavage surgery.

Drug administration. JMT [composed of milkvetch root (Radix Astragali), raw rehmannia root (Radix Rehmanniae), red sage root (Rhizoma Corydalis), kudzuvine root (Herba Asari), leech (Hirudo), dodder seed (Semen Cuscutae), grossy privet fruit (Fructus Ligustri Lucidi), cassia bark tree branchlet (Ramulus Cinnamomi) and Asarum sieboldi] is a medical preparation that is used at the Peking Union Medical College Hospital of the Chinese Academy of Medical Sciences, and is manufactured by Beijing Kowloon Pharmaceutical Co., Ltd. (Beijing, China) (18). Each capsule contained 0.35 g crude drug (lot no. 061019). The rationale (18) behind the use of the various herbs in the JMT formulation according to their use in Chinese medicine is the following: Dodder seed calms yang and nourishes yin of the kidney, secures essence (retains jing or the 'essence' of the kidney), improves vision and alleviates diarrhea; glossy privet fruit nourishes yin of the liver and kidney and clears empty-heat (too much yang or heat pushed up from the kidney); leech and red sage root break blood (improve blood flow), expel stasis and relieve pain; milkvetch root and raw rehmannia root calm qi and nourish yin; cassia twig and kudzuvine root warm and unblock channels and vessels, and promote qi and blood circulation.

As an antioxidant, vitamin $\mathrm{C}$ can improve oxidative stress in diabetics (21), and it was therefore used as a positive control. Vitamin C (0.1 g/tablet) was purchased from Beijing Double-Crane Pharmaceutical Co., Ltd. (Beijing, China; lot no. 080213).

Following model success, the JMT-L group received $0.44 \mathrm{~g} / \mathrm{kg} /$ day JMT, the JMT-M group received $0.88 \mathrm{~g} / \mathrm{kg} /$ day and the JMT-H group received $1.75 \mathrm{~g} / \mathrm{kg} /$ day. These doses were calculated based on the crude drug content of the capsules. The VC group received $0.05 \mathrm{~g} / \mathrm{kg} /$ day vitamin $\mathrm{C}$. These drugs were prepared in distilled water. The DM and Con groups were orally fed distilled water $(10 \mathrm{ml} / \mathrm{kg} /$ day $)$. The rats were treated for 16 weeks. Body weight and tail tip blood glucose levels were determined prior to JMT administration, and at weeks $4,8,12$ and 16 .

Mechanical pain threshold. After 16 weeks of treatment, prior to the sacrifice of the rats, mechanical pain threshold assessment was performed using the von Frey Pain Measurement Instrument (cat. no. 2391; IITC Life Science Inc., Woodland Hills, CA, USA). The rats were placed in an elevated metal net, and covered with transparent organic glass. After a 15-min adaptation period, the middle of the hind foot of each rat was vertically stimulated with the electronic Von Frey probe, making it appear slightly S-shaped, and the paw withdrawal response was observed. A quick flinching reaction immediately subsequent to stimulation was considered to be a positive reaction, and the values (g) were recorded. A paw withdrawal reaction caused by physical activity was not reported as positive.

Sciatic nerve tissue specimen. All rats were sacrificed after 16 weeks of treatment. The rats were intraperitoneally injected with $12 \%$ urethane $(10 \mathrm{ml} / \mathrm{kg})$ and were fixed on the operating table in the prone position. The skin was cut between the biceps femoris and semimembranosus, along the outside line of the femoral shaft and entering into the vastus lateralis muscle. Using glass needles, blunt dissection was performed to expose the sciatic nerve along the two shafts. A total of $2 \mathrm{~cm}$ nerve was fixed using $4 \%$ polyformaldehyde, and the remaining nerve tissue was frozen in liquid nitrogen for polymerase chain reaction (PCR) and western blot analyses.

Reverse transcription-quantitative PCR (RT-qPCR). Total mRNA was extracted from sciatic nerve tissue using TRIzol (Tiangen Biotech Co., Ltd., Beijing, China), according to the manufacturer's instructions. RNA purity was determined using 
Table I. Primers for the reverse transcription-quantitative polymerase chain reaction sequences.

\begin{tabular}{lll}
\hline Gene & \multicolumn{1}{c}{ Primer sequence (5' to 3') } & Product length (bp) \\
\hline$\beta$-actin & Forward: CCCATCTATGAGGGTTACGC & 150 \\
& Reverse: TTTAATGTCACGCACGATTTC & 103 \\
p22 phox & Forward: TATTGTTGCAGGAGTGCTCA \\
& Reverse: CACAGCGGTCAGGTACTTCT \\
Caspase 3 & Forward: TGACTTCTCTCGTCGCTACC & 198 \\
& Reverse: GGTGACATCTCCCTGTTGAC & 158 \\
& Forward: GGACCTGTGGACCTGAAAAA & Reverse: CCGGCATGATGAAAGCGAAGA
\end{tabular}

Bcl-2, B-cell lymphoma 2.

absorbance at 260 and $280 \mathrm{~nm}$ (A260/280). RNA integrity was verified by electrophoresis on formaldehyde gels. Total RNA was reverse-transcribed into cDNAs using oligo (dT) 12-18 primer (Takara, Dalian, China) and Moloney Murine Leukemia Virus reverse transcriptase (Takara).

Primer Express ${ }^{\circledR} 5.0$ software (Applied Biosystems, Foster City, CA, USA) and Oligo 6.0 biological software (Molecular Biology Insights, Inc., Colorado Springs, CO, USA) were used to design the primers shown in Table I. mRNA quantification was performed via qPCR using the BioEasy SYBR Green I Real-Time PCR kit (Bioer Technology Co., Ltd., Hangzhou, China) in a LineGene real-time PCR detection system (Bioer Technology Co., Ltd.). The qPCR reaction program was as follows: Pre-incubation at $95^{\circ} \mathrm{C}$ for $2 \mathrm{~min}$; amplification at $95^{\circ} \mathrm{C}$ for $20 \mathrm{sec}, 59^{\circ} \mathrm{C}$ for $25 \mathrm{sec}$ and $72^{\circ} \mathrm{C}$ for $30 \mathrm{sec}$, for 45 cycles. The melting curve showed increments of $0.5^{\circ} \mathrm{C} / \mathrm{sec}$ between 65 and $95^{\circ} \mathrm{C}$, for a total of $20 \mathrm{sec}$. The threshold cycle $(\mathrm{Ct})$ value was defined as the number of PCR cycles in which the fluorescence signal exceeded the detection threshold value. $\Delta \mathrm{Ct}$ was initially calculated using the equation $\mathrm{Ct}_{\mathrm{Gene}}-\mathrm{Ct}_{\beta \text {-actin }}$, and then $2^{-\Delta \mathrm{Ct}}$ was calculated to represent the relative mRNA expression of the target genes. $\beta$-actin was used as a reference gene.

Immunohistochemistry. Paraffin sections $(5-\mu \mathrm{m})$ were made from sciatic nerves. The sections were dewaxed, washed with $0.01 \mathrm{mmol} / 1$ phosphate-buffered saline (PBS) for $5 \mathrm{~min}$ and boiled in citric acid ( $\mathrm{pH}$ 6.0) for $2 \mathrm{~min}$. Subsequent to cooling, the sections were rinsed three times for $2 \mathrm{~min}$ in $0.01 \mathrm{mmol} / 1$ PBS. Endogenous peroxidase was neutralized with $3 \% \mathrm{H}_{2} \mathrm{O}_{2}$ for $30 \mathrm{~min}$, and the sections were rinsed three times for $2 \mathrm{~min}$ in $0.01 \mathrm{mmol} / \mathrm{l} \mathrm{PBS}$. Bovine serum albumin (3\%) was added and incubated for $20 \mathrm{~min}$ at room temperature. The following primary antibodies were used for incubation at $4^{\circ} \mathrm{C}$, overnight: Rabbit anti-8-OHdG monoclonal antibody (1:100; Japan Institute for the Control of Aging, Nikken SEIL Co., Ltd., Shizuoka, Japan; cat.no. MOG-020P), rabbit anti-NADPH oxidase subunit $\mathrm{p} 22^{\text {phox }}$ polyclonal antibody (1:400; Santa Cruz Biotechnology, Inc., Santa Cruz, CA, USA; cat.no. SC20781), rabbit anti-Bcl-2 polyclonal antibody (1:200; Santa Cruz Biotechnology, Inc.; cat.no. SC783) or rabbit anti-caspase 3 (17 kDa) polyclonal antibody (1:100; Bioworld Technology Inc., Nanjing, China; cat.no. BS7004). The negative control was $0.01 \mathrm{mmol} / \mathrm{l} \mathrm{PBS}$. The sections were rinsed three times for $2 \mathrm{~min}$ in $0.01 \mathrm{mmol} / \mathrm{l} \mathrm{PBS}$ prior to the addition of the secondary antibody (horseradish peroxidase (HRP)-conjugated goat anti-rabbit, cat.no. PV9001; Zhongshan Goldenbridge Biotechnology Co., Ltd., Beijing, China) and incubated at room temperature for $20 \mathrm{~min}$. Subsequent to rinsing three times for $2 \mathrm{~min}$ in $0.01 \mathrm{mmol} / \mathrm{l} \mathrm{PBS}, 50 \mu 1$ 3,3'-diaminobenzidine solution (Zhongshan Goldenbridge Biotechnology Co., Ltd.) was added. The sections were then counterstained with hematoxylin and observed using a Leica DM3000 microscope and the Leica image acquisition system (Leica Microsystems, Wetzlar, Germany). Image-Pro Plus 6.0 software (Media Cybernetics, Inc., Rockville, MD, USA) was used for image analysis. Immunohistochemistry results were measured using the integrated optical density.

Western blot analysis. Total proteins were extracted from the rat sciatic nerves using the Pierce Cell Lysis reagents (Thermo Fisher Scientific, Inc., Waltham, MA, USA) for protein extraction. The resulting solution was centrifuged at $10,000 \mathrm{xg}$ and $4^{\circ} \mathrm{C}$, for $10 \mathrm{~min}$. The bicinchoninic acid (BCA) method (BCA Protein Assay kit; Thermo Fisher Scientific, Inc.) was used to determine the protein concentrations. The same quantity of proteins $(50 \mu \mathrm{g})$ was separated by $6 \%$ SDS-PAGE, and transferred onto polyvinylidene fluoride membranes (GE Healthcare, Waukesha, WI, USA). Non-specific sites were blocked with $5 \%$ powdered milk diluted in Tris-buffered saline with $0.05 \%$ Tween 20 (TBST) for $30 \mathrm{~min}$ at room temperature. Proteins were detected overnight at $4^{\circ} \mathrm{C}$, using the following primary antibodies: Anti-cleaved-PARP-1 $(89 \mathrm{kDa})$ polyclonal antibody (Cell Signaling Technology, Inc., Danvers, MA, USA; cat.no. 9542s) and $\beta$-actin (Sigma, St. Louis, MO, USA; cat.no. A3853). Subsequent to being washed with TBST, the membranes were incubated with HRP-conjugated immunoglobulin G (Zhongshan Goldenbridge Biotechnology Co., Ltd.) for $1 \mathrm{~h}$ at room temperature. The membranes were then further washed with TBST, and the proteins were detected using an enhanced chemiluminescence reagent (Thermo Fisher Scientific, Inc.), according to the manufacturer's instructions, using a FluorChem IS-8800 imaging system (Alpha Innotech Corp., San Leandro, CA, USA). The bands were quantified using the AlphaEaseFC ${ }^{\mathrm{TM}}$ software (Alpha Innotech Corp.). 
Table II. Effect of JMT on fasting blood glucose levels in the diabetic rat model.

Post-treatment (mmol/l)

\begin{tabular}{|c|c|c|c|c|c|c|}
\hline Group & $\mathrm{n}$ & Pre-treatment $(\mathrm{mmol} / \mathrm{l})$ & 4 weeks & 8 weeks & 12 weeks & 16 weeks \\
\hline Con & 10 & $5.0 \pm 0.7$ & $5.1 \pm 0.3$ & $5.4 \pm 0.8$ & $6.1 \pm 0.1$ & $5.4 \pm 0.4$ \\
\hline DM & 11 & $21.1 \pm 3.1^{\mathrm{a}}$ & $21.1 \pm 1.8^{\mathrm{a}}$ & $23.2 \pm 1.0^{\mathrm{a}}$ & $23.9 \pm 2.4^{\mathrm{a}}$ & $21.9 \pm 0.7^{\mathrm{a}}$ \\
\hline JMT-L & 12 & $20.8 \pm 3.2^{\mathrm{a}}$ & $21.2 \pm 1.9^{\mathrm{a}}$ & $22.9 \pm 0.9^{\mathrm{a}}$ & $22.8 \pm 1.6^{\mathrm{a}}$ & $22.3 \pm 0.7^{\mathrm{a}}$ \\
\hline JMT-M & 13 & $21.0 \pm 3.1^{\mathrm{a}}$ & $22.8 \pm 1.7^{\mathrm{a}}$ & $23.5 \pm 0.8^{\mathrm{a}}$ & $23.3 \pm 2.5^{\mathrm{a}}$ & $21.2 \pm 1.8^{\mathrm{a}}$ \\
\hline JMT-H & 12 & $20.8 \pm 3.3^{\mathrm{a}}$ & $22.4 \pm 2.1^{\mathrm{a}}$ & $21.9 \pm 0.7^{\mathrm{a}}$ & $23.4 \pm 1.1^{\mathrm{a}}$ & $22.1 \pm 0.7^{\mathrm{a}}$ \\
\hline $\mathrm{VC}$ & 12 & $21.3 \pm 3.1^{\mathrm{a}}$ & $20.7 \pm 1.4^{\mathrm{a}}$ & $23.8 \pm 0.5^{\mathrm{a}}$ & $23.8 \pm 0.5^{\mathrm{a}}$ & $22.9 \pm 1.1^{\mathrm{a}}$ \\
\hline
\end{tabular}

Data are shown as the mean \pm standard deviation. ${ }^{a} \mathrm{P}<0.01$ vs. Con group. Con, normal control; DM, diabetic model control; JMT, Jinmaitong; -L, -low-dosage; -M, medium-dosage; -H, -high-dosage; VC, vitamin C.

Table III. Effect of JMT on body weight in the diabetic rat model.

Post-treatment $(\mathrm{g})$

\begin{tabular}{|c|c|c|c|c|c|c|}
\hline \multirow[b]{2}{*}{ Group } & \multirow[b]{2}{*}{$\mathrm{n}$} & \multirow[b]{2}{*}{ Pre-treatment (g) } & \\
\hline & & & 4 weeks & 8 weeks & 12 weeks & 16 weeks \\
\hline Con & 10 & $257.2 \pm 20.2$ & $532.5 \pm 10.5$ & $578.3 \pm 24.4$ & $604.1 \pm 33.1$ & $641.2 \pm 15.8$ \\
\hline DM & 11 & $256.4 \pm 17.3$ & $303.6 \pm 10.1^{\mathrm{a}}$ & $321.5 \pm 9.3^{\mathrm{a}}$ & $431.1 \pm 6.8^{a}$ & $468.1 \pm 7.2^{\mathrm{a}}$ \\
\hline JMT-L & 12 & $258.1 \pm 22.1$ & $284.9 \pm 10.1^{\mathrm{a}}$ & $326.5 \pm 10.4^{\mathrm{a}}$ & $426.2 \pm 8.5^{\mathrm{a}}$ & $459.5 \pm 10.7^{a}$ \\
\hline JMT-M & 13 & $256.2 \pm 18.8$ & $303.3 \pm 10.7^{a}$ & $324.5 \pm 10.9^{a}$ & $452.6 \pm 9.3^{\mathrm{a}}$ & $480.6 \pm 12.3^{a}$ \\
\hline JMT-H & 12 & $257.9 \pm 23.9$ & $299.9 \pm 16.6^{\mathrm{a}}$ & $341.7 \pm 14.3^{a}$ & $442.2 \pm 13.6^{\mathrm{a}}$ & $467.8 \pm 14.8^{a}$ \\
\hline $\mathrm{VC}$ & 12 & $257.1 \pm 33.8$ & $301.2 \pm 9.6^{\mathrm{a}}$ & $329.7 \pm 8.3^{\mathrm{a}}$ & $449.2 \pm 10.7^{\mathrm{a}}$ & $477.2 \pm 8.9^{\mathrm{a}}$ \\
\hline
\end{tabular}

Data are shown as the mean \pm standard deviation. ${ }^{\mathrm{P}} \mathrm{P}<0.01$ vs. Con group. Con, normal control; DM, diabetic model control; JMT, Jinmaitong; -L, -low-dosage; -M, medium-dosage; -H, -high-dosage; VC, vitamin C.

Statistical analysis. SPSS 13.0 software (SPSS Inc., Chicago, IL, USA) was used for the statistical analysis. The one-sample Kolmogorov-Smirnov Z-test was used to determine if the variables were normally distributed. Normally-distributed data are expressed as the mean \pm standard deviation. Multi-group independent samples were compared using one-way analysis of variance, with the least significant difference post hoc test. Non-normally-distributed data were analyzed with non-parametric tests. $\mathrm{P}<0.05$ was considered to indicate a statistically significant difference.

\section{Results}

Effect of JMT on fasting blood glucose levels and body weight in the diabetic rat model. Fasting blood glucose levels in all the diabetic rats were higher than those in the control group rats at all time-points (Table II, all $\mathrm{P}<0.01$ ). No significant differences in fasting blood glucose levels were found among the DM, VC and JMT groups at any time-points (Table II, all $\mathrm{P}>0.05)$.

No significant differences in body weight were observed at baseline among the different groups (all $\mathrm{P}>0.05$ ). After 4 weeks of treatment, the body weight of the diabetic rats was lower than that of the control group rats (all $\mathrm{P}<0.01$ ). No significant differences in body weight were found among the DM, VC and JMT groups at any time-points (Table III, all $\mathrm{P}>0.05)$.

Effect of JMT on mechanical pain threshold in the diabetic rat model. The mechanical pain threshold values are listed in Table IV. Compared with the normal controls, the mechanical pain threshold values were significantly lower in the DM group $(\mathrm{P}<0.01)$. The mechanical pain threshold values of each treatment group were higher than those of the DM group (all $\mathrm{P}<0.01$ ). Among the JMT groups, the mechanical pain threshold was significantly higher in the JMT-M group compared with that in the JMT-L, JMT-H and VC groups (all $\mathrm{P}<0.01)$.

Effect of JMT on 8-OHdG and NADPH p22 $2^{\text {phox }}$ expression in the sciatic nerve of the diabetic rat model. The DM group exhibited a 2.1-fold increase in 8-OHdG levels compared with the Con group $(\mathrm{P}<0.01)$. Compared with the DM group, 8-OHdG was decreased in each treatment group (JMT-L, $-11.2 \%$; JMT-M, $-30.0 \%$; JMT-H, $-22.3 \%$ and VC, $-20.2 \%$; all $\mathrm{P}<0.01$ ) (Fig. 1). The decrease in the JMT-M group was more notable than that in the JMT-L, JMT-H and VC groups (all $\mathrm{P}<0.01)$. 
Table IV. Effect of JMT on mechanical pain threshold in the diabetic rat model.

\begin{tabular}{lll}
\hline Group & $\mathrm{n}$ & \multicolumn{1}{c}{ Value (g) } \\
\hline Con & 10 & $78.69 \pm 8.13$ \\
DM & 11 & $35.32 \pm 12.06^{\mathrm{a}}$ \\
JMT-L & 12 & $52.87 \pm 6.46^{\mathrm{a}, \mathrm{b}, \mathrm{c}}$ \\
JMT-M & 13 & $74.69 \pm 9.26^{\mathrm{b}, \mathrm{d}}$ \\
JMT-H & 12 & $61.71 \pm 1.95^{\mathrm{a}, \mathrm{b}, \mathrm{c}}$ \\
VC & 12 & $59.49 \pm 3.42^{\mathrm{a}, \mathrm{b}}$ \\
\hline
\end{tabular}

Data are shown as the mean \pm standard deviation. ${ }^{\mathrm{a}} \mathrm{P}<0.01$ vs. Con group; ${ }^{\mathrm{b}} \mathrm{P}<0.01$ vs. $\mathrm{DM}$ group; ${ }^{\mathrm{c}} \mathrm{P}<0.01$ vs. JMT-M group; ${ }^{\mathrm{d}} \mathrm{P}<0.01 \mathrm{vs}$. VC group. Con, normal control; DM, diabetic model control; JMT, Jinmaitong; -low-dosage; -M, medium-dosage; -H, -high-dosage; VC, vitamin C.

A marked increase in NADPH $\mathrm{p} 22^{\text {phox }}$ mRNA and protein levels was observed in the DM group compared with the Con group (77.6- and 7.6-fold, respectively, both $\mathrm{P}<0.01$ ). The increased NADPH $\mathrm{p} 22^{\text {phox }} \mathrm{mRNA}$ and protein levels in the DM group were then decreased in each treatment group. The results for the protein levels were as follows: JMT-L, -56.1\%; JMT-M, -83.1\%; JMT-H, $-67.8 \%$ and VC, $-41.4 \%$ (all $\mathrm{P}<0.05$ ) (Fig. 2A and B).

Effect of JMT on apoptosis-related gene (Bcl-2, caspase-3 and cleaved-PARP-1) expression in the sciatic nerve of the diabetic rat model. In the DM group, a strong decrease in $\mathrm{Bcl}-2 \mathrm{mRNA}$ and protein levels was induced compared with the Con group (-79.2 and $-87.2 \%$, respectively, both $\mathrm{P}<0.01$ ). Compared with the DM group, the Bcl-2 levels were increased in each treatment group. The results for the protein levels were as follows: JMT-L, 4.0-fold; JMT-M, 4.3-fold; JMT-H, 2.5-fold and $\mathrm{VC}$, 1.3-fold (all $\mathrm{P}<0.01$ ) (Fig. 3A and B). The increase was most evident in the JMT-M group compared with the JMT-L, JMT-H and VC groups (all $\mathrm{P}<0.01$ ).

Caspase 3 mRNA and protein levels in the DM group were markedly increased compared with those in the Con group (11.7- and 5.9-fold, respectively, both $\mathrm{P}<0.01$ ). This increase was then decreased in each treatment group. The results for the protein levels were as follows: JMT-L, $-36.6 \%$; JMT-M, -77.6\%; JMT-H, -48.3\% and VC, 23.9\% (all P<0.01) (Fig. 4A and B).

The DM group exhibited a significant increase in cleaved-PARP-1 levels compared with the Con group (62.6-fold, $\mathrm{P}<0.01)$. The cleaved-PARP-1 levels were decreased in each treatment group compared with those in the DM group (JMT-L, -25.4\%; JMT-M, -62.5\%; JMT-H, -39.4\% and VC, 27.0\%; all $\mathrm{P}<0.05$ ) (Fig. 5). The decrease in the JMT-M group was more notable than that in the JMT-L, JMT-H and VC groups (all $\mathrm{P}<0.01)$.

\section{Discussion}

In the DPN state, there is an imbalance between nerve damage and repair, favoring nerve damage, and leading to decreased
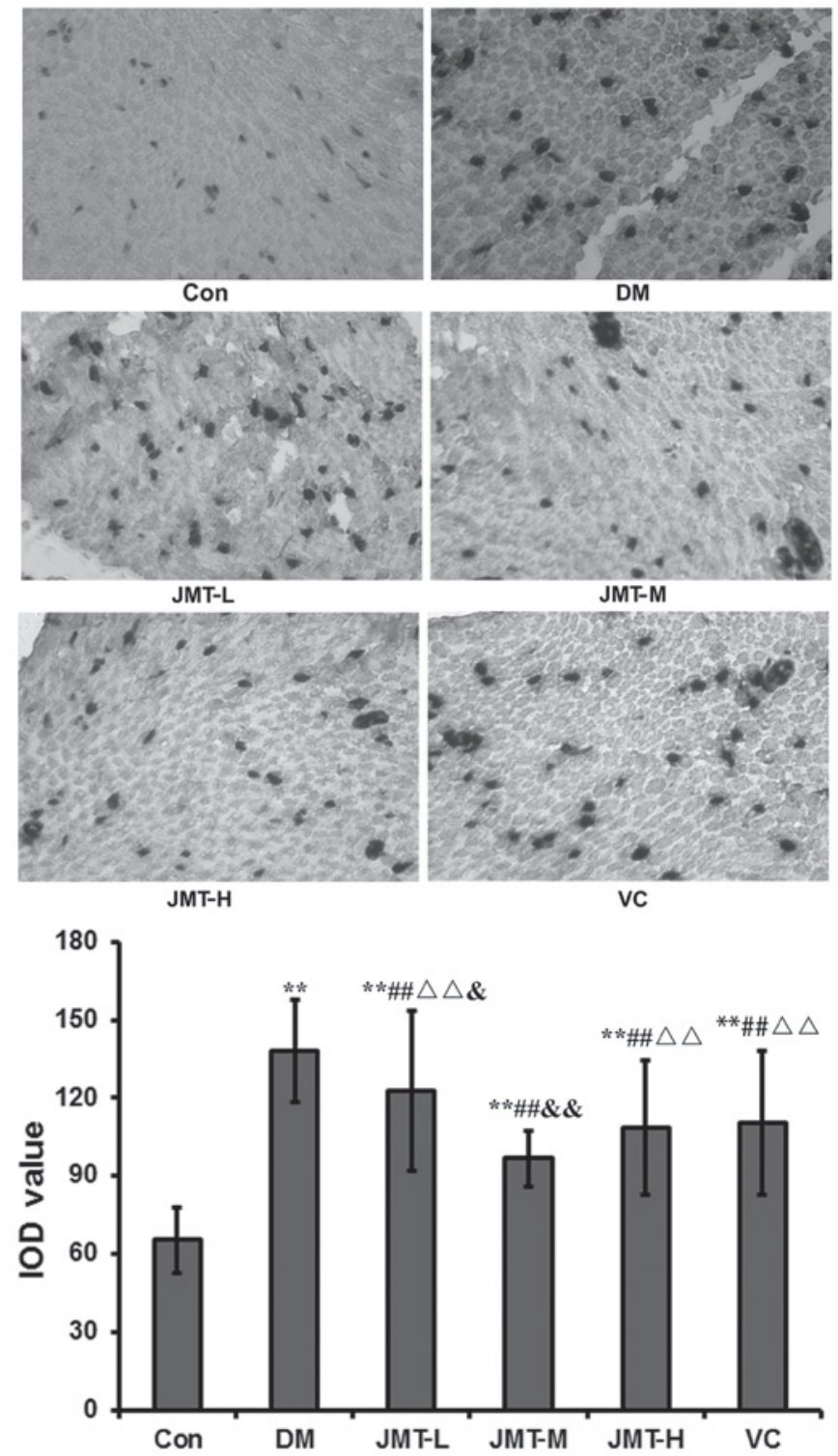

Figure 1. Effect of JMT on 8-OHdG expression in the sciatic nerve in a diabetic rat model. 8-OHdG protein expression was determined by immunohistochemistry (magnification, $x 40$ ). IOD values are shown as the mean \pm standard deviation ( $\mathrm{n}=5$ /group). ${ }^{* *} \mathrm{P}<0.01$ vs. Con; ${ }^{\# \#} \mathrm{P}<0.01$ vs. DM; ${ }^{\Delta} \mathrm{P}<0.01$ vs. JMT-M; ${ }^{\&} \mathrm{P}<0.05$ and ${ }^{\&} \mathrm{P}<0.01$ vs. VC. Con, normal control; DM, diabetic model control; JMT, Jinmaitong; -L, -low-dosage; -M, medium-dosage; -H, -high-dosage; $\mathrm{VC}$, vitamin $\mathrm{C}$; IOD, integrated optical density; 8-OHdG, 8-hydroxy-deoxyguanosine.

sensory perception $(9,10)$; therefore, finding ways to restore this balance is of the prime importance. A number of clinical observations have suggested that JMT could improve limb pain, numbness and other neurological symptoms in patients with DPN $(8,18)$. Furthermore, previous studies have suggested that JMT exerts anti-lipid peroxidation effects $(18,19)$. There is, however, a lack of data regarding the effect of JMT on nerve cells in diabetes.

In the present study it was observed that, following treatment, the rats in the JMT groups had an improved pain threshold compared with the diabetic controls, and the JMT-M group had a pain threshold that was similar to normal control rats. 8-OHdG and NADPH p22 $2^{\text {phox }}$, markers of oxidative DNA damage, were significantly decreased by JMT. Changes in 
A

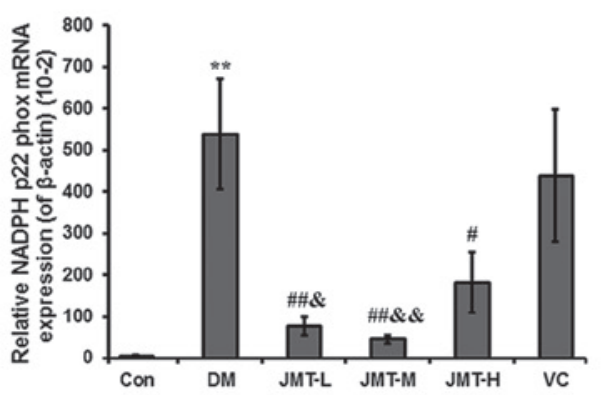

A

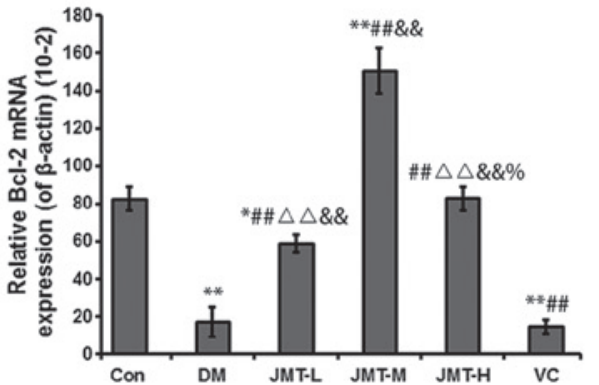

the levels of certain key factors involved in apoptosis, $\mathrm{Bcl}-2$, caspase 3 and cleaved-PARP-1, were all ameliorated by JMT; however, no differences in body weight and fasting blood levels were found among the different diabetic groups, suggesting that JMT does not improve DPN by enhancing diabetes control, but through direct effects on oxidation and nerve cells.

The high glucose levels observed in diabetes increase the levels of reactive oxygen species (ROS) through a number of pathways, intensifying the oxidative stress in all cells, including nerve cells (22). High levels of ROS can lead to DNA damage, which may initiate apoptosis; therefore, any biomarkers of DNA oxidative damage should indicate the level of oxidative stress. NADPH oxidase is an enzyme made up of six subunits, including one $22^{\text {phox }}$ subunit. NADPH oxidase generates a variety of ROS, including the superoxide anion, and measuring the expression of $\mathrm{p} 22^{\text {phox }}$ subunits is a surrogate of NADPH oxidase expression and of ROS production that can attack the DNA (23). When ROS attack the DNA, the guanines at the C- 8 position in the DNA chain can be hydroxylated, thus forming $8-\mathrm{OHdG}$. The presence of 8-OHdG therefore indicates the occurrence of DNA oxidative damage (24). 
A

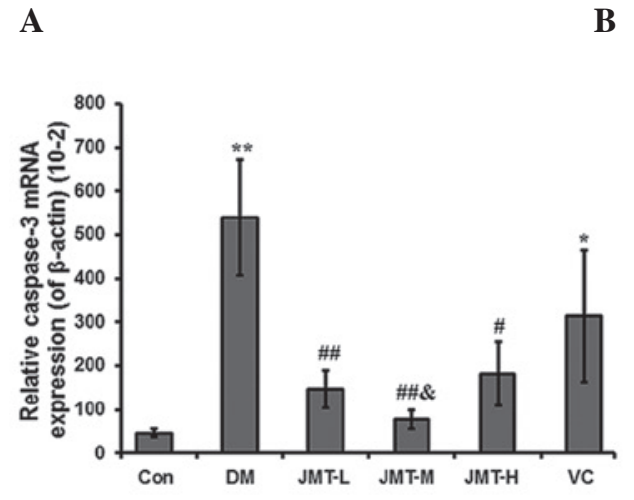

B
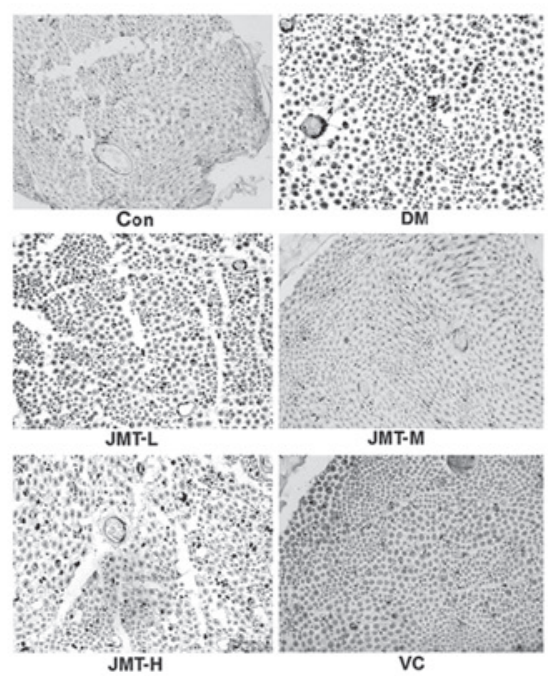

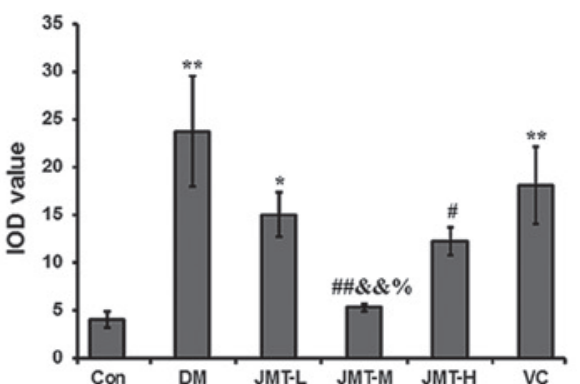

Figure 4. Effect of JMT on caspase-3 mRNA and protein expression in the sciatic nerve in a diabetic rat model. (A) Caspase-3 mRNA expression was determined by reverse transcription-quantitative polymerase chain reaction analysis; $\beta$-actin was used as a reference gene. (B) Active caspase-3 protein expression was determined by immunohistochemistry (magnification, $\mathrm{x} 200$ ). The data are shown as the mean \pm standard deviation ( $\mathrm{n}=5 /$ group). ${ }^{* *} \mathrm{P}<0.01 \mathrm{vs}$. Con; ${ }^{\#} \mathrm{P}<0.05$ and ${ }^{\# \#} \mathrm{P}<0.01$ vs. DM; ${ }^{\&} \mathrm{P}<0.05$ and ${ }^{\& \&} \mathrm{P}<0.01$ vs. VC; ${ }^{\circ} \mathrm{P}<0.05$ vs. JMT-L. Con, normal control; DM, diabetic model control; JMT, Jinmaitong; -L, -low-dosage; -M, medium-dosage; -H, -high-dosage; VC, vitamin C; IOD, integrated optical density.
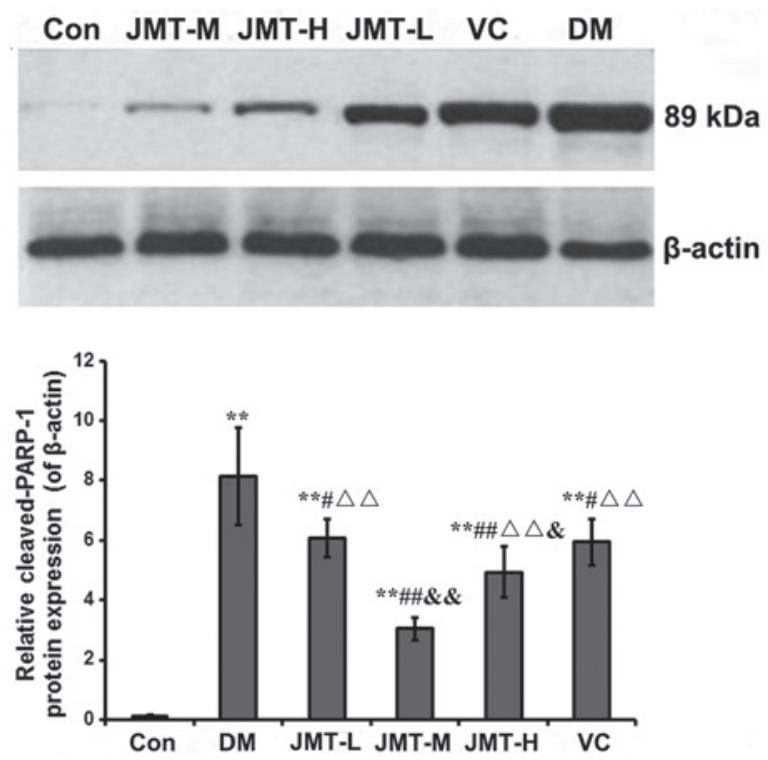

Figure 5. Effect of JMT on cleaved-PARP-1 protein expression in the sciatic nerve in a diabetic rat model. Cleaved-PARP-1 protein expression was determined by western blotting. Protein expression was normalized to $\beta$-actin. IOD values are shown as the mean \pm standard deviation ( $\mathrm{n}=8$ /group). ${ }^{* *} \mathrm{P}<0.01$ vs. Con; ${ }^{\#} \mathrm{P}<0.05$ and ${ }^{\# \#} \mathrm{P}<0.01$ vs. $\mathrm{DM} ;{ }^{\Delta \Delta} \mathrm{P}<0.01$ vs. JMT-M; ${ }^{\circledR} \mathrm{P}<0.05$ and ${ }^{\&} \& \mathrm{P}<0.01$ vs. VC. Con, normal control; DM, diabetic model control; JMT, Jinmaitong; -L, -low-dosage; -M, medium-dosage; -H, -high-dosage; VC, vitamin C; PARP-1, poly(ADP-ribose) polymerase 1.

Following DNA damage, PARP is activated as a DNA damage receptor. PARP-1 is cleaved by caspases, producing the apoptosis-related $24-\mathrm{kDa}$ fragment and the $89-\mathrm{kDa}$ cleaved-PARP-1 fragment. This $89-\mathrm{kDa}$ fragment can be measured as a surrogate of PARP-1 activation $(25,26)$. Cleavage of caspase 3 generates the $17-\mathrm{kDa}$ active subunit, which activates the caspase-activated deoxyribonuclease, which degrades DNA and eventually causes cell death (27). The amount of activated caspase-3 (17 kDa) therefore reflects levels of apoptosis. Bcl-2 is a protein involved in apoptosis regulation and has anti-apoptotic effects through its interactions with a number of factors and pathways (28). As is well known, the diabetic condition shifts these markers towards apoptosis in nerves $(9,10)$, and the present results showed that JMT partly rescues this undesirable shift, as indicated by decreased p22 $2^{\text {phox }}, 8$-OHDG, cleaved-PARP-1 and caspase 3, and by increased Bcl-2.

Using an electronic von Frey instrument to detect the mechanical pain threshold of rats, it was observed in the present study that rats receiving JMT had a better pain threshold than diabetic rats; furthermore, the rats from the JMT-M group had a similar threshold to the normal control rats. The von Frey instrument is commonly used to study pain thresholds in diabetic rat models $(29,30)$, although there is some suggestion that other methods may provide better information on the time and sensitivity of the response with more consistent results (31-33). Despite this, the results suggested that JMT was effective at alleviating hyperalgesia in DPN rats. Since there were no changes in body weight or in the glucose levels of the JMT-treated rats, the results additionally suggested that the effects of JMT on DPN were due to a direct reduction in oxidative stress and apoptosis, rather than a decreased diabetes burden.

A previous study using JMT, in which no changes in body weight and blood levels were observed following JMT treatment, supports the theory that JMT acts by decreasing oxidative stress and apoptosis, and revealed that JMT could improve sciatic nerve health by increasing levels of CNTF, which is involved in a number of survival pathways of nerve cells, favoring their repair (18). The present study suggests that JMT also reduces the oxidative stress on nerve cells in diabetes; however, the exact association between CNTF and oxidative stress in diabetic nerve cells remains to be further explored. JMT has additionally been shown to improve the symptoms of diabetic gastric autonomic neuropathy (18). 
An in vitro study using rat Schwann cells showed that a high-glucose medium inhibited their proliferation, and that JMT had a positive effect by partly restoring cell growth (19). Furthermore, the results of the present study are supported by a previous study in high-glucose-cultured rat Schwann cells showing that JMT increased the expression of Bcl-2 (anti-apoptosis) and decreased the expression of caspase 3 (pro-apoptosis) (34).

These results suggest that JMT has an anti-apoptotic activity that could lead to decreased DPN symptoms, and are consistent with previous studies showing the beneficial effects of antioxidants on DPN $(35,36)$ and with the results using vitamin $\mathrm{C}$. In the present study, however, JMT had a greater effect than vitamin $\mathrm{C}$, and on more parameters. As a complex mix of Chinese herbs JMT is designed to produce a combination of effects and it is only possible to offer speculation regarding which herbs could be responsible for the results observed in this study. Jiangtangshuluofang, which also contains Radix Rehmanniae and Radix Astragali, among other ingredients, has been shown to decrease the apoptosis of Schwann cells (17,37). Although Radix Astragali has been shown to decrease the apoptosis of Schwann cells in isolation, this action increased in combination with other traditional Chinese medicinal herbs $(17,38)$. This indicates that Radix Astragli, or milkvetch root, is at least partially responsible for the apoptosis results. Another point to consider is that the medium dose of JMT produced the most beneficial results in this study. As such, there was not a linear response to the concentration of the dose. This may be due to the highest dose having a detrimental effect. The reasons for this require further investigation, but could be associated with the effects of ROS, such as the immune response, being inhibited.

In conclusion, the results of the present study suggest that the Traditional Chinese Medicine JMT may improve the symptoms of DPN through the inhibition of DNA oxidative damage caused by oxidative stress and by reducing apoptosis in nerve cells from DPN rats. These results suggest that JMT could be a good option to prevent DPN or to improve DPN symptoms in diabetic patients.

\section{References}

1. Dobretsov M, Romanovsky D and Stimers JR: Early diabetic neuropathy: Triggers and mechanisms. World J Gastroenterol 13: 175-191, 2007.

2. Dyck PJ, Kratz KM, Karnes JL, et al: The prevalence by staged severity of various types of diabetic neuropathy, retinopathy and nephropathy in a population-based cohort: The Rochester Diabetic Neuropathy Study. Neurology 43: 817-824, 1993.

3. Tesfaye S: Advances in the management of diabetic peripheral neuropathy. Curr Opin Support Palliat Care 3: 136-143, 2009.

4. Edwards JL, Vincent AM, Cheng HT and Feldman EL: Diabetic neuropathy: Mechanisms to management. Pharmacol Ther 120 $1-34,2008$.

5. Genuth S: Insights from the diabetes control and complications trial/epidemiology of diabetes interventions and complications study on the use of intensive glycemic treatment to reduce the risk of complications of type 1 diabetes. Endocr Pract 12 Suppl 1: $34-41,2006$

6. Vinik A, Emir B, Cheung R and Whalen E: Relationship between pain relief and improvements in patient function/quality of life in patients with painful diabetic peripheral neuropathy or postherpetic neuralgia treated with pregabalin. Clin Ther 35: 612-623, 2013.

7. Nicholson B: Differential diagnosis: Nociceptive and neuropathic pain. Am J Manag Care 12 (Suppl): S256-S262, 2006.
8. Shakher J and Stevens MJ: Update on the management of diabetic polyneuropathies. Diabetes Metab Syndr Obes 4: 289-305, 2011.

9. Russell JW, Sullivan KA, Windebank AJ, Herrmann DN and Feldman EL: Neurons undergo apoptosis in animal and cell culture models of diabetes. Neurobiol Dis 6: 347-363, 1999.

10. Allen DA, Yaqoob MM and Harwood SM: Mechanisms of high glucose-induced apoptosis and its relationship to diabetic complications. J Nutr Biochem 16: 705-713, 2005.

11. El Boghdady NA and Badr GA: Evaluation of oxidative stress markers and vascular risk factors in patients with diabetic peripheral neuropathy. Cell Biochem Funct 30: 328-334, 2012.

12. Figueroa-Romero C, Sadidi M and Feldman EL: Mechanisms of disease: The oxidative stress theory of diabetic neuropathy. Rev Endocr Metab Disord 9: 301-314, 2008.

13. Kasznicki J, Kosmalski M, Sliwinska A, et al: Evaluation of oxidative stress markers in pathogenesis of diabetic neuropathy. Mol Biol Rep 39: 8669-8678, 2012.

14. Schmeichel AM, Schmelzer JD and Low PA: Oxidative injury and apoptosis of dorsal root ganglion neurons in chronic experimental diabetic neuropathy. Diabetes 52: 165-171, 2003.

15. Sun LQ, Zhao J,Zhang TT, et al: Protective effects of Salvianolic acid B on Schwann cells apoptosis induced by high glucose. Neurochem Res 37: 996-1010, 2012.

16. Hernández-Beltrán N, Moreno CB and Gutiérrez-Álvarez AM: Contribution of mitochondria to pain in diabetic neuropathy. Endocrinol Nutr 60: 25-32, 2013.

17. Piao $\mathrm{Y}$ and Liang $\mathrm{X}$ : Chinese medicine in diabetic peripheral neuropathy: Experimental research on nerve repair and regeneration. Evid Based Complement Alternat Med 2012: 191632, 2012

18. Shi Y, Liang XC, Wu QL, et al: Effects of Jinmaitong Capsule on ciliary neurotrophic factor in sciatic nerves of diabetes mellitus rats. Chin J Integr Med 19: 104-111, 2013.

19. Qu L, Liang XC, Zhang H, Wu QL, Sun LQ and Gu B: Effect of Jinmaitong serum on the proliferation of rat Schwann cells cultured in high glucose medium. Chin J Integr Med 14: 293-297, 2008.

20. Kim J, Yokoyama K and Araki S: The effects of Ginkgo biloba extract $(\mathrm{GBe})$ on axonal transport microvasculature and morphology of sciatic nerve in streptozotocin-induced diabetic rats. Environ Health Prev Med 5: 53-59, 2000.

21. Varvarovská J, Racek J, Stetina R, et al: Aspects of oxidative stress in children with type 1 diabetes mellitus. Biomed Pharmacother 58: 539-545, 2004.

22. Pop-Busui R, Sima A and Stevens M: Diabetic neuropathy and oxidative stress. Diabetes Metab Res Rev 22: 257-273, 2006.

23. Bedard K and Krause KH: The NOX family of ROS-generating NADPH oxidases: Physiology and pathophysiology. Physiol Rev 87: 245-313, 2007.

24. Toyokuni S: Reactive oxygen species-induced molecular damage and its application in pathology. Pathol Int 49: 91-102, 1999.

25. Obrosova IG, Xu W, Lyzogubov VV, et al: PARP inhibition or gene deficiency counteracts intraepidermal nerve fiber loss and neuropathic pain in advanced diabetic neuropathy. Free Radic Biol Med 44: 972-981, 2008.

26. Oliver FJ, de la Rubia G, Rolli V, Ruiz-Ruiz MC, de Murcia G and Murcia JM: Importance of poly(ADP-ribose) polymerase and its cleavage in apoptosis. Lesson from an uncleavable mutant. J Biol Chem 273: 33533-33539, 1998.

27. Lamkanfi M, Festjens N, Declercq W, Vanden Berghe T and Vandenabeele P: Caspases in cell survival, proliferation and differentiation. Cell Death Differ 14: 44-55, 2007.

28. Qin W, Hu J, Guo M, et al: BNIPL-2, a novel homologue of BNIP-2, interacts with Bcl-2 and Cdc42GAP in apoptosis. Biochem Biophys Res Commun 308: 379-385, 2003.

29. Fuchs D, Birklein F, Reeh PW and Sauer SK: Sensitized peripheral nociception in experimental diabetes of the rat. Pain 151: 496-505, 2010.

30. Dobretsov M, Hastings SL, Romanovsky D, Stimers JR and Zhang JM: Mechanical hyperalgesia in rat models of systemic and local hyperglycemia. Brain Res 960: 174-183, 2003.

31. Vivancos GG, Verri WA Jr, Cunha TM, et al: An electronic pressure-meter nociception paw test for rats. Braz J Med Biol Res 37: 391-399, 2004

32. Chaplan SR, Bach FW, Pogrel JW, Chung JM and Yaksh TL: Quantitative assessment of tactile allodynia in the rat paw. J Neurosci Methods 53: 55-63, 1994.

33. Farghaly HS, Abd-Ellatief RB, Moftah MZ, Mostafa MG, Khedr EM and Kotb HI: The effects of dexmedetomidine alone and in combination with tramadol or amitriptyline in a neuropathic pain model. Pain Physician 17: 187-195, 2014. 
34. Wang PY, Liang XC, Zhang H, et al: Effect of serum containing Jinmaitong Capsule on rats' Schwann cell apoptosis induced by high glucose concentration. Chin J Integr Med 19: 517-523, 2013.

35. Pazdro R and Burgess JR: The role of vitamin E and oxidative stress in diabetes complications. Mech Ageing Dev 131: 276-286, 2010.

36. Vincent AM, Edwards JL, Sadidi M and Feldman EL: The antioxidant response as a drug target in diabetic neuropathy. Curr Drug Targets 9: 94-100, 2008.
37. Ji JL and Chen DS: Experiment study of nourishing yin, invigorating qi, extinguishing wind, promoting blood circulation and draining collateral method of the sciatic nerve Schwann cells apoptosis of diabetic rats. Zhongguo Zhong Yi Ji Zheng 18: 1304-1306, 2009 (In Chinese).

38. Liu XY, Zhang YY and Zhang JQ: Effect of astragalus, salvia, yam and its compound on apoptosis of Schwann cells co-cultured with endothelial cells in high glucose. Zhong Yao Yao Li Yu Lin Chuang 26: 41-44, 2010 (In Chinese). 